\title{
Lidil
}

Revue de linguistique et de didactique des langues

\section{Une approche active de la morphographie}

L'exemple d'une séquence sur l'accord de l'adjectif

\section{Danièle Cogis}

\section{(2) OpenEdition}

\section{Journals}

Édition électronique

URL : http://journals.openedition.org/lidil/743

DOI : $10.4000 /$ lidil. 743

ISSN : 1960-6052

Éditeur

UGA Éditions/Université Grenoble Alpes

\section{Édition imprimée}

Date de publication : 1 décembre 2004

Pagination : 73-86

ISBN : 2-914176-11-2

ISSN : $1146-6480$

Référence électronique

Danièle Cogis, " Une approche active de la morphographie », Lidil [En ligne], 30 | 2004, mis en ligne le

29 janvier 2008, consulté le 04 mai 2019. URL : http://journals.openedition.org/lidil/743 ; DOI :

10.4000/lidil.743

(c) Lidil 


\section{UNE APPROCHE ACTIVE DE LA MORPHOGRAPHIE \\ L'EXEMPLE D'UNE SÉQUENCE SUR L'ACCORD DE L'ADJECTIF}

\section{Danièle CoGIS *}

L'acquisition de l'écrit est un des objectifs fondamentaux de l'école et l'orthographe en est une composante importante, qui intervient dans la longueur et la qualité des écrits des élèves (Graham, 1990). Étant donné le poids et la spécificité de la morphographie en français, il n'est pas étonnant que les difficultés se concentrent sur cette dimension du plurisystème graphique : c'est là que se situent les plus vives tensions entre les composantes phonographique et sémiographique de notre orthographe (Catach, 1990; Jaffré, 1997).

Des recherches menées depuis plus d'une décennie, qu'elles soient de type expérimental (Brissaud, 1998) ou fondées suì des entretiens métagraphiques (Jaffré, 2003), ont permis de cerner les procédures des élèves et la dynamique de leur évolution. Comme les travaux d'Emilia Ferreiro l'ont montré pour le principe alphabétique, les élèves élaborent la morphographie à leur manière (Bousquet et al., 1999 ; Cogis, 2002; Sandon, 1999).

En regard, l'enseignement traditionnel de l'orthographe grammaticale, fondé sur des règles (leçons) et des rappels de règles (corrections), apparait bien peu adapté : à l'école ou au début du collège, la plupart des élèves ne disposent pas encore des connaissances qui leur permettraient d'appliquer ces règles, et certaines de leurs conceptions font obstacle à la pro-

* IUFM de Paris - LEAPLE (Université Paris 5 - UMR 8606, CNRS). 
duction de la norme. Afin de répondre à des contraintes aujourd'hui mieux identifiées, il est donc nécessaire d'opter pour d'autres démarches.

Cet article se propose de montrer, dans les perspectives ouvertes par Vygotski et Bruner, que certaines séquences d'apprentissage peuvent conduire les élèves à une meilleure conceptualisation de la morphographie. On prendra l'exemple de l'accord de l'adjectif afin d'illustrer quelques aspects de l'appropriation d'un savoir réputé élémentaire.

\section{Une réputation en question}

L'adjectif fait partie de ces notions grammaticales que l'on dit volontiers simples, au point que son accord est évalué au bout de deux ans d'apprentissage systématique de l'écrit. Comme pour d'autres notions, il n'est jamais expliqué ce qui fonde un tel choix, sinon, on peut le supposer, qu'il existe une règle d'accord et que l'ajout d'une lettre $(e, s)$ parait un geste simple: c'est sans compter la quantité de connaissances nécessaires à la «simple » production de ces morphogrammes hautement polyvalents (Cogis, 2001). Après le verbe et son sujet (Cogis et Brissaud, 2003), il a semblé intéressant d'aller voir de près la relation nom/adjectif que doivent connaitre les écoliers, sinon au début du cycle 3 , du moins à la fin.

- Une première question s'impose: que sait-on de la maitrise de l'accord de l'adjectif par les élèves? Les évaluations nationales de $6^{\mathrm{e}}$ en donnent une certaine idée.

En s'en tenant à un exercice régulièrement proposé, où il s'agit de réécrire, avec les accords appropriés, un texte dont on a modifié le sujet thématique, les résultats peuvent sembler faibles à l'issue de l'école primaire (adjectifs, participes employés comme adjectifs ou avec être confondus) ${ }^{1}$ :

1. Pour 1990, 1991, 1992, MEN, DEP, Education \& Formations, Évaluations CE2 - 6*, Hors série (1991): 132; (1992): 152-155; (1992): 21, 162-165. Pour 1998, 1999 et 2000, 2001, MEN, DPD, Dossiers Évaluations CE2 - 6 $,(1999): 111,174-175$; (2001): 124, 224-225; (2002): 128, 204-205. 
- moins d'un quart des élèves accorde un ensemble de 4 unités (montées, seules, étonnées, petites, 1990); moins du tiers accorde les 3 items d'un groupe nominal, nom inclus (de grands guerriers, pleins, 2001);

- moins de la moitié des élèves accorde 2 ou 3 adjectifs d'une série qui en comporte 3 (noires, indifférentes, majestueuses, 1998); moins de la moitié un adjectif sur deux (grands, pleins, 2001).

Quant aux 16 items dont le score est singularisé, leur taux de réussite moyen est de $64,8 \%$ (mais s'étend de 49,2 à $81,7 \%$, avec une moyenne de $79,6 \%$ pour les trois meilleurs vs $49,8 \%$ pour les trois plus faibles). Là encore, les résultats sont loin de correspondre aux attentes de l'institution pour qui certains de ces accords devraient déjà être maitrisés trois ans plus tôt.

Partant de là, l'habitude est de déplorer ou de s'indigner, au lieu de prendre avec sérénité ces résultats pour ce qu'ils sont: un état du niveau de connaissances des élèves réel et non imaginaire à la fin de l'école; et d'en tirer les indications pour lesquelles ces évaluations nationales ont été prévues: l'apprentissage de l'accord des adjectifs/participes dans des chaines de plusieurs unités est à poursuivre au collège.

- Une seconde question se pose alors: comment peut-on expliquer les écarts de réussite entre items?

Au-delà de ces données, en effet, ce qui est intéressant, c'est le score obtenu par 7 items proposés à deux ou trois reprises de 1990 à 2001. Il se situe toujours dans une même «région» de l'échelle: trempées et glissées, avec 5 occurrences, prennent les 5 premières places; les 4 occurrences d'indifférentes et de majestueuses les 4 suivantes; impressionnés et enthousiastes se placent du $11^{\mathrm{e}}$ au $14^{\mathrm{e}}$ rang. Seules, les 2 occurrences de noires se dispersent un peu entre le $10^{\mathrm{e}}$ et le $15^{\mathrm{e}}$ rang.

Cette homogénéité dans les résultats ne peut donc s'expliquer par un simpliste «Ils connaissent les règles mais ne les appliquent pas». Elle est bien davantage le reflet de ce qui différencie les items, pourtant soumis aux mêmes règles d'accord, à savoir des caractéristiques linguistiques - graphiques, morphologiques, syntaxiques, sémantiques ou pragmatiques. 
En effet, approches linguistiques et psycholinguistiques se rejoignent pour souligner que la catégorie adjective n'est pas uniforme, mais s'organise en prototypes et satellites (Noailly, 1999) ou en plus ou moins bons candidats à l'«adjectivité» (François et al., 1986). N'est-ce pas sur quoi bute cette élève de CM2 qui ne sait pas, en fonction de ses critères définitoires du moment, si malpolie est un adjectif, alors qu'elle n'a aucun doute pour «pauvre, petit, très courants à l'écrit» (Cogis, 2002)?

Des études ont pointé certains facteurs pour expliquer l'inégale reconnaissance ou production des accords: la longueur de la chaine d'accord, la visibilité du signal déclencheur, la présence de rupteurs, ces éléments qui s'intercalent entre la source et la cible de l'accord (Jaffré et Bessonnat, 1993); mais aussi le type d'adjectif (qualifiant/classifiant), la fonction syntaxique (épithète/attribut), la position par rapport au nom (antéposé/postposé) et le caractère mono- ou transcatégoriel de l'unité (Fisher, 2001); pour le nombre, le rapport marque/classe syntaxique (Thévenin et al., 1999) ou encore, pour le genre, la structure morphologique (Cogis, 2002). Or, pour pouvoir appliquer la règle d'accord à un adjectif, il faut d'abord l'identifier comme appartenant à la classe des adjectifs avec toutes les contraintes afférentes: on va voir que ce repérage pose problème.

De fait, les items des évaluations nationales présentent des disparités formelles (grands vs noires, par exemple). On peut alors sans doute attribuer leur inégale réussite aux procédures différentes que leurs caractéristiques linguistiques suscitent et, par-delà, à une plus ou moins grande difficulté conceptuelle pour des élèves de 11 ou 12 ans. Là où l'expert semble produire des marques d'accord sans distinction, eux se montrent très dépendants des circonstances, sans parler de leurs capacités de traitement et de contrôle. Comment faire alors en classe?

\section{Une séquence d'orthographe en CM2}

En fin de cycle 3, la règle d'accord de l'adjectif n'est pas un sujet neuf. Mais les élèves ne savent pas la mettre en œuvre de 
façon systématique, car ils ne perçoivent pas toujours les liens syntaxiques ou substituent une autre logique à celle attendue. Il importe donc de les amener à confronter leurs raisonnements aux principes de la morphographie dans toutes sortes de configurations linguistiques.

Un dispositif possible est la phrase dictée du jour qui fait discuter les élèves de leurs graphies, produites lors de la dictée d'une phrase, puis notées au tableau, afin de déterminer laquelle est la bonne; ce dispositif est parfois complété par une étude de corpus (Cogis, 2003; 2004). Le travail présenté s'est déroulé en quatre séances dans un CM2 de bon niveau ${ }^{2}$. On en résume ici l'essentiel.

\section{Séance 1 : impasse}

Rusées, les sœurs de Cendrillon lui donnent de longs travaux à faire.

de l'on travaux
long traveaux
longs
lon
longe
lomg

Lors d'une première phase, les élèves éliminent longe, l'on, lon, le premier pour sa prononciation, les autres grâce au féminin longue; lomg est rapproché de longtemps dont le $m$ semble avoir été à l'origine de celui de lomg; la construction de longtemps ayant été analysée, lomg est effacé. La discussion a donc permis de mettre en pièces toutes les propositions qui ne respectent pas l'identité graphique de l'unité long: le morphogramme lexical $g$ et à un moindre degré le digramme on semblent bien traduire aux yeux des élèves le lien qu'ils perçoivent entre signifié et signifiant dans toute la famille lexicale.

Si le débat porte surtout sur les graphies constitutives du mot, deux justifications du pluriel sont cependant avancées,

2. Merci à J. Hardy-Vulbeau, formatrice, à ses élèves et aux professeurs-stagiaires de l'IUFM de Paris qui ont participé à ce travail en janvier 2003. 
l'une par Léa: «Les sœurs, elles donnent plusieurs travaux à faire à Cendrillon, alors... »; l'autre, plus explicite, par Anne : «Au féminin, ça s'écrit longue, $\mathrm{j}$ 'ai pensé qu'il y avait un $g$ et parce qu'il y a plusieurs travaux, donc $\mathrm{j}$ 'ai mis un $s »$. Mais c'est surtout quand les graphies qui dénaturent le mot sont éliminées (« Ça peut pas s'écrire $l$ '-o-n ou $l-o-n$, parce que c'est pas un mot lon, et aussi ben parce qu'il y a pas de $g$ à la fin») qu'il devient plus facile de reprendre la question. La seconde phase de la discussion est alors presque entièrement consacrée aux deux graphies qui restent en lice, long et longs. La classe se divise en deux camps.

\section{- Long}

Chez les minoritaires qui défendent long, on distingue trois lignes d'arguments. D'abord l'habituel «Je le vois écrit comme ça». Ensuite, le sémantisme de long, perçu comme un ensemble continu qui convoque le singulier: «On met tous les travaux ensemble, alors c'est très long». Cette idée possède une grande force puisque, vers la fin du débat et malgré le poids des arguments en faveur du pluriel, elle sera reprise par Gaëtan: "Mais peut-être qu'il peut y avoir une règle sur $l-o-n-g$ et que la règle dit tout le temps $l-o-n-g »$. Enfin, une partie des élèves ne parvient pas à construire de longs travaux comme un syntagme pluriel parce qu'ils ne disposent pas d'un signal initial connu. Comme finit par le dire Maria: «Parce que, si on dit des longs travaux, ben là on peut le garder, mais si on dit de longs... tra... vaux... ». Même en l'état, l'argument renforce la conviction de la minorité.

\section{- Longs}

Pour la majorité, l'idée de pluriel, bien présente, n'est cependant pas identique. Pour les uns, classiquement, il y a pluriel parce qu'il y a plusieurs travaux à faire. Et la classe de s'engager dans une discussion sur le nombre et la durée des travaux, qui aboutit à cet argument irrécusable de Claire: «Elle va pas en faire qu'un seul, parce que sinon à la limite, elle pourrait aller au bal».

La confrontation entre partisans du singulier et partisans du pluriel amène ainsi les élèves à réfléchir aux indéfinis, à leur sens, et plus particulièrement au statut de de. Par exemple 
avec Clélia qui s'oppose aux tenants du singulier: «Mais de et des, ça revient un peu au même ! », ou Anne : «C'est quand même du pluriel», leurs modalisations gardant la trace du paradoxe conceptuel ou de leur conviction nouvelle.

\section{- De longs travaux}

Parallèlement au pluriel référentiel, ce qui passe progressivement au premier plan chez d'autres élèves, c'est la dimension formelle. En réalité, dès la quatrième minute de discussion, Siméon avait fait état des rapports syntagmatiques: «C'est longs, parce que... travaux déjà, c'est au pluriel, parce que sinon on dirait un travail, et donc c'est obligé qu'il y ait un $s$ », argument aussitôt contesté : «Non, c'est pas obligé».

Ce n'est que dans la seconde phase du débat, après la série des justifications sémantico-référentielles qu'Anne récupère l'argument : «Si c'était au singulier, à la place de de, on dirait un long travail au lieu de de longs travaux, donc... avec un $s »$. Rachel prend le relai en appuyant sur l'opposition un long travail/de longs travaux. Calypso observe: «De, ça peut être aussi au pluriel, parce que de longs travaux, quand on dit travaux, c'est... enfin normalement, au singulier, c'est travail, donc ça veut dire que de, ça peut aussi faire le pluriel». Siméon martèle que c'est au pluriel parce qu'on ne peut dire de long travail, mais, de nouveau, l'argument est récusé. C'est au tour de Clélia d'insister: «Long... c'est pas possible parce que, un, c'est au singulier, alors que travaux, c'est plusieurs travaux...».

Le raisonnement sous-jacent est à présent d'une tout autre envergure. Il ne porte plus sur la possibilité que de ou long soient ou non au pluriel, mais sur la solidarité syntagmatique entre les éléments d'un groupe: à partir du moment où l'un d'entre eux est un pluriel - et travaux l'est indiscutablement les autres le sont nécessairement.

Cependant, la concordance morphosyntaxique ne prend pas force de loi auprès de tous les élèves. L'enseignante décide alors de laisser la question en suspens pour la retravailler. La discussion globale a duré plus de douze minutes, sans déboucher sur un accord. Néanmoins, la controverse est si forte qu'elle se poursuit sur le palier! 


\section{Séance 2 : résolution}

Pour sortir de l'impasse, l'enseignante met en place une séance d'un autre type. Les élèves, après avoir rappelé les arguments en présence, qui sont notés au tableau, doivent commenter toutes les occurrences de long dans un mini-corpus de cinq phrases (un long voyage vs de longues distances). La classe fait alors deux constats: de correspond bien à un pluriel; long a toujours le même nombre que le nom.

Une synthèse collective sur «L'adjectif qualificatif dans le groupe nominal » élargit la question à l'ensemble de la classe syntaxique; elle est ensuite consignée dans le cahier des phrases du jour, qui sert de répertoire morphosyntaxique, avec la glose plusieurs travaux qui sont longs en regard du syntagme discuté.

\section{Séance 3 : confirmation}

Les remous qui agitent la classe trois jours plus tard lors d'une nouvelle phrase dictée, au moment où Maria annonce qu'elle a écrit de long cheveux, montrent que les élèves ont reconnu le cas de figure. Elle-même dit qu'elle s'est trompée. Mais la classe n'a plus le temps d'en débattre.

\section{Séance 4: vérification}

Quiñze jours après, les élèves se voient proposer dans de profonds tunnels. Dès le début de la discussion, Sabine annonce son erreur (profond) dans une formulation quelque peu ambivalente: "J'ai cru que c'était un tunnel... Et à la place de $s$, j'ai mis un $d$... Parce qu'ils disent dans de profonds tunnels, donc de, c'est plusieurs, je pense ». L'argument a encore une résonance référentielle, mais c'est bien l'équivalence entre de et des qui est affirmée d'entrée de jeu, alors qu'elle était niée la première fois, même avec la preuve du pluriel travaux. Quant à ce qui est présenté à tort comme une substitution entre $d$ et $s$, la remarque témoigne que les deux morphogrammes de nature différente commencent à être pensés conjointement.

Basile, de son côté, approfondit la réflexion sur la valeur du déterminant de, à la fois indéfini et pluriel : «Dans le pro- 
fond tunnel, dans de profonds tunnels, ça veut dire qu'il y en a plusieurs, c'est pas un tunnel précis». Pour Alex, cependant, le statut de de, s'il s'éclaircit, est encore flou, comme le montre sa réponse: «De, pour toi ça signifie plusieurs? Non, pas vraiment, mais quand j'entends dans de, c'est un peu plusieurs ». Finalement, Basile, même s'il ne parvient pas encore à le faire de façon rigoureuse, amorce le paradigme un/de: "Alors si le, ça désigne un, de, ça veut dire plusieurs", équivalence immédiatement matérialisée par l'enseignante au tableau. Le pluriel profonds en sort consolidé.

\section{L'apprentissage de la morphographie}

L'apprentissage de la morphographie comporte nécessairement la découverte de son fonctionnement, mais cela ne suffit manifestement pas. Si un adjectif aussi prototypique que long pose autant de problèmes à des élèves de CM2 de bonne volonté, certains allant même jusqu'à admettre de long travail, on comprend mieux les résultats des évaluations nationales. Il fautalors travailler à un autre niveau, celui des représentations et des procédures, dans une séquence de plusieurs séances, construite en fonction des problèmes qui se manifestent, pour que des apprentissages aient lieu, comme ici sur l'accord de l'adjectif.

\section{Apprentissages linguistiques}

Dans cette séquence, indubitablement, il s'agit d'apprentissage. D'une part, les contenus notionnels sont bien des contenus linguistiques, travaillés avec les opérations de commutation et de transformation: le groupe nominal, l'accord de l'adjectif, la fonction lexicale ou grammaticale des lettres finales, leur enchainement, la notion de famille et de construction de mots, sans oublier la découverte du déterminant de. D'autre part, une évolution s'est produite. Elle se lit dans les chiffres: de 17 à ne pas marquer le pluriel la première fois (de longs travaux), les élèves ne sont plus que 5 (de longs cheveux), puis 7 (de profonds tunnels) à l'omettre ensuite, et 
ce, malgré la disparition des repères facilitateurs (reprise de longs ou pluriel phonique travaux). La diminution de la durée des débats est aussi intéressante parce qu'elle indique une maturation collective: de 12 minutes pour un constat de désaccord, on passe à 1 pour dénoncer l'omission du pluriel, puis à 6 pour fonder solidement la présence des morphogrammes $d$ et $s$. Quant à la durée globale de la séquence, elle est plus que raisonnable: 1 h 30 environ répartie sur 4 séances. Et, surtout, on note des changements qualitatifs essentiels pour l'acquisition de la morphographie: plus personne n'allègue le sémantisme de long pour s'opposer au pluriel; ceux qui refusaient de comme un signal de pluriel l'admettent ; des procédures morphosyntaxiques (commutation de/un, le) cœxistent plus nettement avec les procédures morphosémantiques encore très répandues à cet âge.

L'enjeu d'une telle séquence d'orthographe n'est donc pas tant de "corriger» long ou profond en répétant la règle, mais de faire élaborer le syntagme nominal comme un groupe dont tous les membres sont morphologiquement solidaires, en amenant les élèves à passer du particulier au général par des opérations linguistiques (aucun cependant ne reprend jamais le terme adjectif, indice d'un passage à la généralisation).

Ainsi, pour assimiler la notion d'accord de l'adjectif, les élèves doivent dépasser une approche phono/logographique (tel mot, telle suite de lettres) pour s'approprier la variation et en saisir les principes (tels morphogrammes dans tel contexte) sans se laisser piéger par une approche morphosémantique trop centrée sur la quantité référentielle - en bref, s'engager dans le monde parfois déroutant de la morphosyntaxe.

Ce qui rend possible cette avancée, ou du moins la favorise, c'est le cadre structuré de la démarche qui fournit un étayage à l'activité mentale des élèves.

\section{Paramètres d'une démarche}

Cette séquence d'orthographe se caractérise par le rôle joué par les interactions verbales, le conflit sociocognitif et l'observation de corpus.

Les interactions verbales sont en effet au cœur du processus: pris par le défi de convaincre les autres, les élèves sont 
obligés de verbaliser leurs façons de faire. C'est donc l'écart entre la tâche (trouver la bonne graphie) et l'activité (mobiliser des connaissances) qui offre aux élèves cet espace de pensée et de confrontation indispensable à la construction des savoirs par le langage (Nonnon, 1996). Comme avec le déterminant leur au singulier (Cogis, 2003), tout se passe comme si les élèves devaient produire une série de reformulations pour se convaincre que l'adjectif s'accorde toujours avec le nom (l'intérêt de de, déterminant du registre soutenu, est bien de révéler la fragilité actuelle de l'accord de l'adjectif). La progression des connaissances est laborieuse, au sens qu'elle nécessite du labeur, un vrai travail de la pensée qui n'a rien à voir avec cet «écoute et applique» sur lequel échoue l'enseignement traditionnel de l'orthographe avec trop d'élèves. Ici, les reformulations successives, sous leur apparente redondance, font circuler des points de vue comme dans une charade, et finalement construisent des savoirs. Par exemple, une élève pointe le morphogramme lexical, une autre le morphogramme grammatical, une troisième reprend le tout. Les élèves les moins avancés apprennent ainsi des autres comment se traite la morphographie; les plus avancés affinent leurs raisonnements et leurs formulations.

Mais les élèves ne se laissent pas toujours convaincre et le débat se bloque. Comme un argument d'autorité peut faire obstacle à la réélaboration conceptuelle nécessaire, mieux vaut alors recourir à un corpus normé - objet tiers - pour que les élèves appréhendent ce qu'ils ne parvenaient pas à concevoir. Le conflit n'est d'ailleurs pas toujours spectaculaire, le simple énoncé d'un autre point de vue suffit à créer une faille dans une conception spontanée dont on perçoit les effets plus tard (Perret-Clermont et Nicolet, 1988; Cogis, 2004).

Ici, la loi syntagmatique semble d'autant mieux (r)établie qu'elle a été combattue, signe probable d'une activité mentale située dans la zone de développement potentiel. Conforme aux nouveaux programmes du cycle 3 , le travail proposé, bien loin du rappel impuissant des règles, a permis aux élèves, en combinant observations de leurs graphies, situations de résolution de problème, analyse de corpus normé, synthèse, d'opérer des prises de conscience nécessaires au développement de la morphographie et des futurs automatismes. 
Pour adopter des procédures requises par l'univers de la morphographie, les élèves doivent rompre avec le principe phono/logographique et assimiler des fonctionnements complexes (polyvalence des graphèmes, structures syntaxiques) tâche difficile dont l'enseignement ne peut se décharger, en ignorant que les élèves «réinterprètent» dans leurs propres cadres de pensée le savoir transmis.

C'est le but d'une séquence de ce type que de répondre à cette obligation. Si on leur propose un étayage pertinent, au lieu de les culpabiliser pour des fautes qui ne sont souvent que le reflet d'un niveau de compréhension du système, les élèves sont capables de progresser en s'engageant réellement dans la réflexion métalinguistique. Dans cette perspective, la confrontation avec autrui et avec les objets culturels que sont les formes linguistiques de l'écrit jouent un rôle déterminant. On peut alors penser qu'un autre rapport à la langue a des chances de s'instaurer.

\section{Références bibliographiques}

Bousquet, S., Cogis, D., Ducard, D., Massonnet, J., JafFRÉ, J.-P. (1999): Acquisition de l'orthographe et mondes cognitifs, Revue française de pédagogie, 126, 23-37.

BRISSAUD, C. (1998): Acquisition de l'orthographe du verbe au collège: le cas des formes en /E/. Invariants et procédures, Thèse de doctorat, Université Stendhal-Grenoble 3.

CATACH, N. (1990): L'écriture en tant que plurisystème, ou théorie de L prime, in N. Catach (éd), Pour une théorie de la langue écrite. Actes de la Table Ronde internationale CNRS-HESO, 23-24 octobre 1986, Éditions du CNRS.

CoGIS, D. (2001): Difficultés en orthographe: un indispensable réexamen, Revue française de linguistique appliquée, VI-1, 47-61.

CoGIS, D. (2002): Comment le genre graphique vient aux enfants, in G. Haas (éd.), Apprendre, comprendre l'orthographe autrement de la maternelle au lycée, Scéren-CRDP de Bourgogne, 19-41. 
CogIs, D. (2003): Construction des connaissances, langage et orthographe: vers un nouveau contrat didactique, in M. Jaubert, M. Rebière, J.-P. Bernié (éds), Construction des connaissances et langage dans les disciplines d'enseignement, Actes du Colloque pluridisciplinaire international, Bordeaux, avril 2003, CD-Rom.

CogIs, D. (2004) : Dire l'orthographe pour l'acquérir, in Fautil parler pour apprendre? Actes du colloque, Arras, mars 2004, CD-Rom.

Cogis, D., Brissaud, C. (2003) : L'orthographe: une clé pour l'observation réfléchie de la langue ? Repères, 28, 47-70.

FisHeR, C. (2001): Manifestations des représentations grammaticales d'élèves du primaire dans deux tâches, in J. Dolz, B. Schneuwly, T. Thevenaz-Christen, M. Wirthner (éds), Les tâches et leurs entours en classe de français, Actes $8^{e}$ colloque DFLM 2001, CD-Rom.

François, F., CNockaert, D., Leclerc, S. (1986): Noms, verbes et adjectifs ou définir et classer, Études de linguistique appliquée, 62, 26-39.

Graham, S. (1990): The Role of Production Factors in Learning Disabled Students'Compositions, Joumal of Educational Psychology, Vol. 82, 4, 781-791.

J.AFFRE, J.-P. (1997): Des écritures aux orthographes: fonctions et limites de la notion de système, in L. Rieben, M. Fayol, C. Perfetti (éds), Des orthographes et leur acquisition, Delachaux et Niestlé, 19-36.

JAFFRÉ, J.-P. (2003): Les commentaires métagraphiques, Faits de langues, 22, 67-76.

JAFFRÉ, J.-P., BESSONNAT, D. (1993): Accord ou pas d'accord. Les chaines morphologiques, Pratiques, 77, 25-42.

NoAILly, M. (1999): L'adjectif en français, Gap, Paris, Ophrys.

NoNNON, É. (1996): Activités argumentatives et élaboration de connaissances nouvelles: le dialogue comme espace d'exploration, Langue française, 112, 67-87.

Perret-Clermont, A.-N., Nicolet, M. (1988): Interagir et connaitre. Enjeux et régulations sociales dans le développement cognitif, rééd. 2001, Paris, L'Harmattan. 
SANDON, J.-M. (1999): Les unités linguistiques et leurs frontières: statut et fonctions dans l'acquisition de l'orthographe du français, Thèse de Doctorat, Paris 3.

Thévenin, M.-G., Totereau, C., Fayol, M., Jarousse, J.-P. (1999): L'apprentissage/enseignement de la morphologie écrite du nombre en français, Revue française de pédagogie, 126, 39-52. 